\title{
Glatiramer Acetate, Dimethyl Fumarate, and Monomethyl Fumarate Upregulate the Expression of CCR10 on the Surface of Natural Killer Cells and Enhance Their Chemotaxis and Cytotoxicity
}

OPEN ACCESS

Edited by:

Nurit Hollander,

Tel Aviv University, Israel

Reviewed by:

Norberto Walter Zwirner,

Institute of Biology and Experimental Medicine (IBYME-CONICET),

Argentina

Jacques Zimmer

Centre de Recherche Public de la

Santé, Luxembourg

Estrella Mariel Levy,

National Scientific and Technical Research Council, Argentina

*Correspondence:

Azzam A. Maghazachi amagazachi@sharjah.ac.ae

Specialty section:

This article was submitted to Immunotherapies and Vaccines,

a section of the journal

Frontiers in Immunology

Received: 14 August 2016 Accepted: 04 October 2016 Published: 19 October 2016

Citation:

Maghazachi AA, Sand KL and Al-Jaderi Z (2016) Glatiramer Acetate, Dimethyl Fumarate, and Monomethyl Fumarate Upregulate the Expression of CCR10 on the Surface of Natural

Killer Cells and Enhance Their

Chemotaxis and Cytotoxicity.

Front. Immunol. 7:437.

doi: 10.3389/fimmu.2016.00437

\section{Azzam A. Maghazachi ${ }^{1 *}$, Kristin L. Sand ${ }^{2}$ and Zaidoon Al-Jaderi ${ }^{1,2}$}

${ }^{1}$ Department of Clinical Sciences, College of Medicine, and the Sharjah Institute for Medical Research (SIMR), University of Sharjah, Sharjah, United Arab Emirates, ${ }^{2}$ University of Oslo, Oslo, Norway

In vitro harnessing of immune cells is the most important advance in the field of cancer immunotherapy. Results shown in the current paper may be used to harness natural killer (NK) cells in vitro. It is observed that drugs used to treat multiple sclerosis such as glatiramer acetate, dimethyl fumarate, and monomethyl fumarate upregulate the expression of chemokines receptor 10 (CCR10) on the surface of human IL-2-activated NK cells. This is corroborated with increased chemotaxis of these cells toward the concentration gradients of the ligands for CCR10, namely CCL27 and CCL28. It is also demonstrated that these three drugs enhance NK cell cytotoxicity against tumor target cells, an activity that is abrogated by prior incubation of the cells with anti-CCR10 antibody. Because CCL27 and CCL28 are secreted by selective tumor types such as malignant melanoma, squamous cell carcinomas, and colorectal cancer, respectively, it is hypothesized that activated NK cells may be harnessed in vitro with any of these drugs before utilizing them as a therapeutic modality for cancer.

Keywords: NK cells, glatiramer acetate, dimethyl fumarate, monomethyl fumarate, cancer, chemotaxis, cytotoxicity

\section{INTRODUCTION}

Natural killer (NK) cells perform several important functions; among them, the regulation of the adaptive immune response by secreting cytokines such as IFN- $\gamma$, shaping the innate immune system by interacting with dendritic cells, defending against viral infection, and lysing and destroying tumor cells (1-5). Resting NK cells respond to dangers occurring at sites of injury. Evidence gathered from a mouse xenograft tumor model testing functionally deficient NK cells or antibody-mediated NK cell depletion supports that NK cells can eradicate tumor cells. An 11-year follow-up study in patients indicated that low NK-like cytotoxicity was associated with increased cancer risk (6). High levels of tumor-infiltrating NK cells are associated with a favorable tumor outcome in patients with colorectal carcinoma, gastric carcinoma, and squamous cell lung cancer, suggesting that NK cell infiltration into tumor tissues represents a positive prognostic marker (7). 
Chemokines are molecules that play essential roles in linking the innate and adaptive immune responses (8) and are crucial in health and diseases (9). They have low molecular weights and are divided into four subfamilies based on the position of the cysteine $(\mathrm{C})$ residue in the amino terminal end of the molecules; these are known as CXC, CC, C, and CX3C. They play important roles in $\mathrm{NK}$ cell biology maintaining them in the bone marrow, guiding them into the circulation, and aiding their accumulation at sites of injury. The field examining the expression of chemokine receptors in NK cells, and their ability to induce their migration, started in early 1990 s $(10,11)$. There are overwhelming results describing the effects of chemokines on various biological activities of NK cells [reviewed in Ref. (12)].

Glatiramer acetate (GA; commercial name Copaxone) is a synthetic compound made up of the four amino acids Glu, Ala, Lys, and Tyr that are most common in myelin basic protein (13). GA is a first-line immunomodulatory therapy in relapsing-remitting multiple sclerosis (RRMS) patients (14). Although the drug is not as effective as second-line therapies such as Natalizumab and Fingolimod, GA is widely used due to few serious side effects. This drug showed promise in maintenance therapy, when used after more intensive immunosuppression (15). It was also reported that GA enhanced the in vitro killing of autologous and allogeneic human immature and mature monocyte-derived dendritic cells (DCs) by activated human NK cells (16). Further, administration of GA into mice ameliorated the EAE clinical scores, and this was associated with high killing of dendritic cells by NK cells isolated from the same mice (17).

Dimethyl fumarate (DMF), also known as Tecfidera (Biogen, Cambridge, MA, USA), has been approved by the FDA as an oral therapy for multiple sclerosis (MS) patients due to its efficacy. The mechanism of action of DMF is not completely understood. However, it was suggested that DMF may be hydrolyzed by esterases to monomethyl fumarate (MMF), although it is not yet clear whether MMF might mediate the in situ effects of DMF (18). It has also been demonstrated that DMF inhibits the proliferation of A375 or M24met cell lines and reduces melanoma growth and metastasis in experimental melanoma mouse models (19).

We recently reported that MMF increased primary human $\mathrm{CD}^{2} 6^{+} \mathrm{NK}$ cell lysis of K562 and RAJI tumor cells (20). Moreover, MMF upregulated the expression of NKp46 on the surface of NK cells, which was correlated with upregulation of CD107a (lysosomal-associated membrane protein-1 "LAMP-1") on the surface of $\mathrm{CD}^{2} 6^{+} \mathrm{NK}$ cells, and the release of Granzyme B from CD56 NK cells (20). Moreover, MMF inhibited the EAE clinical score in SJL mice correlated with enhanced NK cell lysis of dendritic cells (21).

In the present report, we describe a novel effect of GA, MMF, and DMF. We observed that these drugs upregulate the expression of CCR10 on the surface of IL-2-activated NK cells, corroborated with increased cytotoxicity, and induced chemotaxis toward the ligands for CCR10, namely CCL27 and CCL28. These observations may have implications for utilizing the highly antitumor effector NK cells in the therapy of cancer, particularly for those patients where tumor cells secrete the ligands for CCR10.

\section{MATERIALS AND METHODS}

\section{Reagents}

FITC-conjugated mouse antihuman CCR3, CCR4, CCR5, CCR6, CCR7, CCR9, CXCR1, CXCR3, CXCR4, and CXCR5 or unconjugated monoclonal mouse-antihuman CCR1, CCR2, and CXCR6, as well as PE-conjugated rat antihuman CCR8, PE-conjugated rat antihuman CCR10, and PE-conjugated rat IgG2b, were obtained from R\&D Systems Europe Ltd. (Abingdon, UK). FITC-conjugated mouse antihuman $\mathrm{CX}_{3} \mathrm{CR} 1$ was purchased from Medical and Biological Laboratories Co. Ltd. (Nagoya, Japan). FITC-conjugated monoclonal mouse antihuman CD3, PE-conjugated monoclonal mouse antihuman CD56, and FITC-conjugated goat anti-mouse were purchased from BectonDickinson (San Diego, CA, USA). FITC-conjugated mouse IgG, PE-conjugated mouse IgG, unconjugated mouse IgG, and unconjugated rat IgG were obtained from either Becton-Dickinson or from R\&D Systems. Pertussis toxin (PTX), MMF, and DMF were obtained from Sigma-Aldrich (Saint Louis, MO, USA). CCL1, CCL27, CCL28, and CXCL10 were purchased from PeproTech (London, UK).

\section{Preparation and Culture of NK Cells}

Buffy coats from normal human volunteers were obtained from the blood bank (Ulleval Hospital, Oslo). Human IL-2-activated NK cells were prepared using Histopaque-1077 (Sigma-Aldrich) and RosetteSep human NK cell enrichment cocktail (Stemcell Technologies, SARL, Grenoble, France). NK cells were negatively selected by removing cells expressing CD3, CD4, CD19, CD36, CD66b, CD123, and glycophorin A. More than 95\% of these cells expressed the CD56 molecule but lacked the CD3 molecule as determined by flow cytometric analysis (Figure S1 in Supplementary Material). Purified NK cells were then placed in flasks at $1 \times 10^{6} / \mathrm{mL}$ and $200 \mathrm{U} / \mathrm{mL}$ IL-2 (PeproTech, Rocky Hill, $\mathrm{NJ}, \mathrm{USA}$ ), and then incubated at $37^{\circ} \mathrm{C}$ in a $5 \% \mathrm{CO}_{2}$ incubator for 5-7 days.

\section{NK Cell Cytotoxicity Assay}

The human myeloid leukemia cell line K562 cells (CCL-243 obtained from American type culture collection "ATCC," Manassas, VA, USA) or RAJI human lymphoma cells (CCL-214, ATCC), were used as target cells. Target cells were incubated at $1 \times 10^{6}$ cells $/ \mathrm{mL}$ with $5 \mu \mathrm{g} / \mathrm{mL}$ Calcein AM (Sigma-Aldrich) for $45 \mathrm{~min}$. The cells were pelleted by centrifugation and resuspended in RPMI. To obtain total lysis, these cells were incubated in 96-well plates with $0.05 \%$ Triton X, whereas they were incubated with medium alone to obtain total viability. In other cultures, Calcein-AM-labeled cells were incubated at $37^{\circ} \mathrm{C}$ in a $5 \% \mathrm{CO}_{2}$ incubator with activated NK cells at different $\mathrm{NK}$ target cell ratios for $4 \mathrm{~h}$. The plates were centrifuged, supernatants were removed, and replaced with PBS. Fluorescence units (FUs) were measured in Cytofluor plate reader. The percentage of cytotoxicity was calculated according to the following formula: $\%$ viability $=\mathrm{FU}$ of targets incubated with IL-2-activated NK cells (experimental) minus FU of targets incubated with Triton X, divided by FU of targets incubated in medium only (total viability), minus FU of 
targets incubated with Triton X (total lysis). In the figures, the 10:1 effector:target (E:T) cell ratio is shown; however, similar results were obtained using other E:T cell ratios (2.5:1 and 5:1). Of note, the media of incubation contains RPMI plus 10\% FCS. For anti-CCR10 treatment, the cells were washed and then treated with $10 \mu \mathrm{g} / \mathrm{mL}$ anti-CCR10 or $10 \mu \mathrm{g} / \mathrm{mL}$ isotype control antibody for $45 \mathrm{~min}$ at $4^{\circ} \mathrm{C}$, washed and examined for viability. Only more than $95 \%$ viable cells were then added to Calcein-AM-labeled target cells and incubated for $4 \mathrm{~h}$ in the NK cytotoxicity assay.

\section{In Vitro Chemotaxis Assay}

Nucleopore blind well chemotaxis chambers with a lower well volume of $200 \mu \mathrm{L}$ were used. A maximum volume of $200 \mu \mathrm{L}$ medium containing RPMI plus 2\% FCS was placed in the lower wells in the presence or absence of various chemokines. Cells $\left(2 \times 10^{5}\right)$ were placed in the upper compartments and incubated for $2 \mathrm{~h}$ at $37^{\circ} \mathrm{C}$ in a $5 \% \mathrm{CO}_{2}$ incubator separated by polycarbonate filters (Nucleopore Polycarbonate $13 \mathrm{~mm}$ size $8 \mathrm{UM}$, Whatman International Ltd., Kent, UK). Filters were removed, dehydrated, stained with $15 \%$ modified Giemsa stain for $7 \mathrm{~min}$, and then mounted on glass slides. Cells in 10 high power fields were counted and averaged for each sample. Migration index (MI) was calculated as the number of cells migrating toward the concentration gradients of chemokines divided by the number of cells migrating toward medium only as previously described (22). For pretreatment with PTX, NK cells $\left(1 \times 10^{6} / \mathrm{mL}\right)$ were either left intact or were treated for $2 \mathrm{~h}$ at $37^{\circ} \mathrm{C}$ with $100 \mathrm{ng} / \mathrm{mL}$-activated PTX, as previously described (22). Only more than 95\% viable cells were examined.

\section{Flow Cytometric Analysis}

IL-2-activated NK cells were either left intact or incubated with various concentrations of GA, MMF, or DMF for $24 \mathrm{~h}$. The cells were washed and incubated in a 96-well plate (v-bottom, $2 \times 10^{5}$ cells per well), washed again, and resuspended in PBS buffer containing $0.1 \%$ sodium azide and $2 \%$ fetal bovine serum. Cells were first checked for viability with Trypan blue exclusion test, and only more than $95 \%$ viable cells were used in the assay. These viable cells were labeled with antibodies for $45 \mathrm{~min}$ in the dark at $4^{\circ} \mathrm{C}$, washed twice, and examined in the flow cytometer (FACSC II, Becton-Dickinson Biosciences, San Jose, CA, USA). Markers were set according to the isotype control FITC- or PE-conjugated mouse IgG. More than 95\% viable NK cells were also incubated at $4^{\circ} \mathrm{C}$ in the dark with FITC-conjugated anti-CD107a or FITC-conjugated IgG1 isotype control (BectonDickinson Pharmingen, San Diego, CA, USA). They were washed twice and examined in the flow cytometry. Gating was performed according to the isotype control. Analysis was done by FlowJo (Flow cytometry analysis software, Ashland, OR, USA).

\section{Cell Lysis and Immunoblotting}

More than 95\% viable NK cells treated with $100 \mathrm{ng}, 1 \mu \mathrm{g}$, or $10 \mu \mathrm{g}$ GA for $24 \mathrm{~h}$ were lysed with ice-cold Non-idet P-40 lysis buffer containing 1\% Non-idet P-40, 30 mM Tris-HCl, pH 7.4, $150 \mathrm{mM}$ $\mathrm{NaCl}, 10 \mathrm{mM}$ NaF, $1 \mathrm{mM}$ EDTA, $10 \mathrm{mM}$ sodium pyrophosphate, $1 \mathrm{mM}$ sodium orthovanadate, $1 \mathrm{mM}$ phenylmethyl-sulfonyl fluoride (PMSF), and a protease inhibitor cocktail (Sigma-Aldrich).
The solutions were centrifuged at $13,000 \times g$ for 15 min at $4^{\circ} \mathrm{C}$ to separate the cell lysates from the supernatants. Total protein concentration in the samples was determined using Bio-Rad Protein Assay (Bio-Rad, Uppsala, Sweden), and samples with $20-\mu \mathrm{g}$ protein were used for each well in the gel. The samples were run on SDS-PAGE criterion gels and then electro transferred to PVDF-membranes (Millipore, Bedford, MA, USA). The membranes were blocked with 5\% skim milk in TBS for $1 \mathrm{~h}$, and then incubated overnight at $4^{\circ} \mathrm{C}$ with 1:250 dilution of polyclonal rabbit antihuman CCR10 or 1:8000 dilution of polyclonal rabbit antiGADPH (Both from Abcam, Cambridge, UK). The membranes were washed four times in TBS-T-buffer, before incubation with HRP-conjugated secondary antibody (1:2500) (Bio-Rad) for $1 \mathrm{~h}$ at room temperature. Binding of antibodies to the target proteins was detected by secondary HRP-labeled antibodies and Super Signal West Pico Stable Peroxide Solution (Pierce, Rockford, IL, USA), using chemiluminescence film and CURIX 60 (Agfa HealthCare, Mortsel, Belgium).

\section{Detection of CCL27 (CTACK) and CCL28 (MEC) by ELISA}

Natural killer cells $\left(1 \times 10^{6} / \mathrm{mL}\right)$ were incubated with $100 \mathrm{ng}$, $1 \mu \mathrm{g}$, or $10 \mu \mathrm{g}$ of $\mathrm{GA}$, or with culture medium as a control for $24 \mathrm{~h}$ at $37^{\circ} \mathrm{C}$ in $5 \% \mathrm{CO}_{2}$ incubator. After incubation, the cells were harvested, and the cell suspensions were centrifuged at $1000 \mathrm{~g}$ for $8 \mathrm{~min}$. Supernatants were collected and stored at $-80^{\circ} \mathrm{C}$ until further analysis. Levels of chemokine were measured using ELISA kits from RayBiotech Inc. (Norcross, GA, USA). Color intensity was measured at $450 \mathrm{~nm}$ in a BioTek PowerWave XS plate reader. The standard curves and concentrations were calculated using Gen5 Data Analysis Software (BioTek Instruments, Winooski, VT, USA).

\section{Detection of Granzyme B by Flow Cytometric Analysis}

IL-2-activated NK cells $\left(1 \times 10^{6} / \mathrm{mL}\right)$ were left intact for $24 \mathrm{~h}$. Supernatants were collected from these cells and were kept at $-80^{\circ} \mathrm{C}$ until use. To detect the expression of Granzyme B (GrB), the cells $\left(1 \times 10^{6} / \mathrm{mL}\right)$ were incubated with either media alone or with $10 \mu \mathrm{g} / \mathrm{mL}$ of GA overnight. In addition, the cells treated as above (with or without GA) were preincubated with supernatants collected from activated NK cells either alone or with $1 \mu \mathrm{g} / \mathrm{mL}$ mouse antihuman CCL27 or mouse antihuman CCL28 (R\&D systems), and as a control mouse IgG1.

To stain the intracellular GrB, cells were incubated with $10 \mu \mathrm{g} / \mathrm{mL}$ Brefeldin A (Sigma-Aldrich) for $4 \mathrm{~h}$. They were then fixed with $4 \%$ paraformaldehyde for $15 \mathrm{~min}$ at $4^{\circ} \mathrm{C}$ and then washed twice with SAP buffer (PBS with $0.1 \%$ Saponin and $0.05 \%$ $\mathrm{NaN} 3$ ) before staining intracellularly with either PE-conjugated mouse antihuman Granzyme B or isotype control PE-conjugated mouse IgG antibody (both from ImmunoTools, Friesoythe, Germany) in the dark at $4^{\circ} \mathrm{C}$ for $45 \mathrm{~min}$. Cells were washed with flow cytometric medium and resuspended with PBS in 5-mL tubes to perform flow cytometric analysis. Gating was done according to the PE-conjugated isotype control antibody. Analysis was done by FlowJo (flow cytometry analysis software, Ashland, OR, USA). 


\section{Statistical Analysis}

Significant values were generated using several tests. We used one-way ANOVA, two-way ANOVA with Bonferroni post hoc correction, or the Student's $t$-test. A $P$ value $<0.05$ was considered to be statistically significant.

\section{RESULTS}

\section{GA Upregulates the Expression of CCR10 on the Surface of IL-2-Activated NK Cells and Induces Their Chemotaxis Toward \\ Its Ligands}

Natural killer cells migrate into inflammatory sites aided by sets of chemokine receptors, which direct them toward the chemokines present at tumor growth sites. To this end, we investigated the expression of CCR1, CCR2, CCR3, CCR4, CCR5, CCR6, CCR7, CCR8, CCR9, CCR10, CXCR1, CXCR2, CXCR3, CXCR4, CXCR5, CXCR6, CXCR7, and $\mathrm{CX}_{3} \mathrm{CR} 1$ on the surface of IL-2activated NK cells after $24 \mathrm{~h}$ incubation with 1 or $10 \mu \mathrm{g} / \mathrm{mL}$ of GA. Only more than $90 \%$ viable cells as determined by Trypan blue exclusion test were used in this and subsequent assays. The reasons for choosing activated NK cells are due to the fact that at, inflammatory sites such as the tumor microenvironment, inflammatory molecules including cytokines and chemokines are released, which activate NK cells. In addition, activated NK cells migrate toward sites of inflammation much more efficiently than non-activated cells [reviewed in Ref. (12)].

From all the chemokine receptors examined, GA upregulated the expression of CCR1 and CCR10 on the surface of activated NK cells (Figure 1A). Also shown are histograms where CCR10 is expressed on activated NK cells after incubating overnight with $10 \mu \mathrm{g} / \mathrm{mL}$ of GA (Figure 1B). We focused the rest of this work on the expression of CCR10, since another drug used for treating MS patients, namely DMF also upregulates similar expression (also see Figure 4). To confirm the upregulation of CCR10 after GA stimulation, we performed immunoblot analysis. Results in Figure 1C demonstrate that overnight incubation with $0.1,1$, or $10 \mu \mathrm{g} / \mathrm{mL}$ of GA induced the expression of this receptor on activated NK cells, despite the fact that flow cytometric analysis did not show increase in expression of this receptor after incubation with $1 \mu \mathrm{g} / \mathrm{mL}$ of GA (Figure 1A).

Consequently, we examined the chemotaxis of activated NK cells treated for $24 \mathrm{~h}$ with $10 \mu \mathrm{g} / \mathrm{mL}$ GA toward various concentrations (ranging from 1 to $1000 \mathrm{ng} / \mathrm{mL}$ ) of CCL27 and CCL28, the ligands for CCR10. Cells untreated with GA migrated with low intensity toward high concentrations of CCL27 (100 and $1000 \mathrm{ng} / \mathrm{mL}$ ) and CCL28 (10 and $100 \mathrm{ng} / \mathrm{mL}$ ), which could be due to the low number of CCR10 positive NK cells. However, cells treated with GA for $24 \mathrm{~h}$ migrated toward much lower concentrations of CCL27 and CCL28 (Figure 1D). When compared to untreated cells, the migration of cells toward the lower concentrations of these chemokines was enhanced by twofold to fivefold after treatment with GA, an enhancement that was statistically significant (Figure 1D). As a control, we also observed that these cells migrated toward the concentration gradients of CCL3, one of the ligands for CCR1 after treatment with GA (Figure 1D), which is corroborated with increased CCR1 expression on the surface of these cells after similar treatment.

\section{Pertussis Toxin Inhibits the Chemotaxis of NK Cells}

We also investigated whether $G$ proteins might be involved in mediating the chemotactic response. Consequently, activated NK cells were either left intact or were pretreated with PTX for $2 \mathrm{~h}$, which intoxicates and inhibits the function of $\mathrm{G}_{\mathrm{i}} / \mathrm{G}_{\mathrm{o}}(22)$. As shown in Figure 2A, NK cells migrated toward $10 \mathrm{ng} / \mathrm{mL}$ CCL27 after preincubating for $24 \mathrm{~h}$ with $10 \mu \mathrm{g} / \mathrm{mL}$ GA $(P<0.001$, as compared to the control). This effect was inhibited after preincubating NK cells with PTX $(P<0.04)$. Similarly, $1 \mathrm{ng} / \mathrm{mL}$ of CCL28 induced the chemotaxis of NK cells pretreated overnight with GA $(P<0.04$, as compared to the control), and this activity was abrogated upon pretreating the cells with PTX $(P<0.05$, Figure 2B).

\section{GA Enhances NK Cell Lysis of Tumor Cells: Inhibition by Anti-CCR10 Antibody}

To evaluate whether GA might induce activities other than chemotaxis in NK cells, we performed the cytotoxicity assay. Results shown in Figure 3 demonstrate that incubating NK cells overnight with $10 \mu \mathrm{g} / \mathrm{mL}$ of this drug increased activated NK cell lysis of K562 cells (Figure 3A), or RAJI cells (Figure 3B). Because GA upregulates the expression of CCR10 on the surface of NK cells, we sought to demonstrate whether such expression might influence the cytolytic activity of these cells. NK cells either untreated or incubated with $10 \mu \mathrm{g} / \mathrm{mL}$ anti-CCR10, or as a control with isotype control IgG antibody, were examined for their ability to lyse tumor target cells in the NK cytotoxicity assay. We observed that anti-CCR10, and not the isotype control IgG, tended to inhibit NK cell lysis induced by GA of K562 (Figure 3A), but significantly inhibited lysis of RAJI cells $(P<0.05$, comparing killing in the presence of anti-CCR10 to isotype control IgG, Figure 3B). Of note, cells treated overnight with GA and then incubated with isotype control IgG antibody also lysed K562 cells (Figure 2A) or RAJI cells (Figure 2B), but this did not reach statistical significance when compared to untreated cells incubated with the isotype control antibody.

\section{IL-2-Activated NK Cells Secrete CCL27 and CCL28}

Because anti-CCR10 inhibited GA-induced NK cell cytotoxicity, we entertained various possibilities to explain such activity. First, we sought to determine whether GA might induce the release of CCL27 or CCL28 from activated NK cells, and that these chemokines might facilitate NK cell cytolytic activity by binding CCR10. Our results demonstrate that IL-2-activated NK cells released an average of $280 \mathrm{pg} / \mathrm{mL}$ of CCL27 (Figure S2A in Supplementary Material), or 500 pg/mL CCL28 (Figure S2B in Supplementary Material). However, addition of various concentrations ranging from 0.1 to $10 \mu \mathrm{g} / \mathrm{mL}$ of GA to IL-2-activated $\mathrm{NK}$ cells did not significantly affect the levels of these chemokines, 


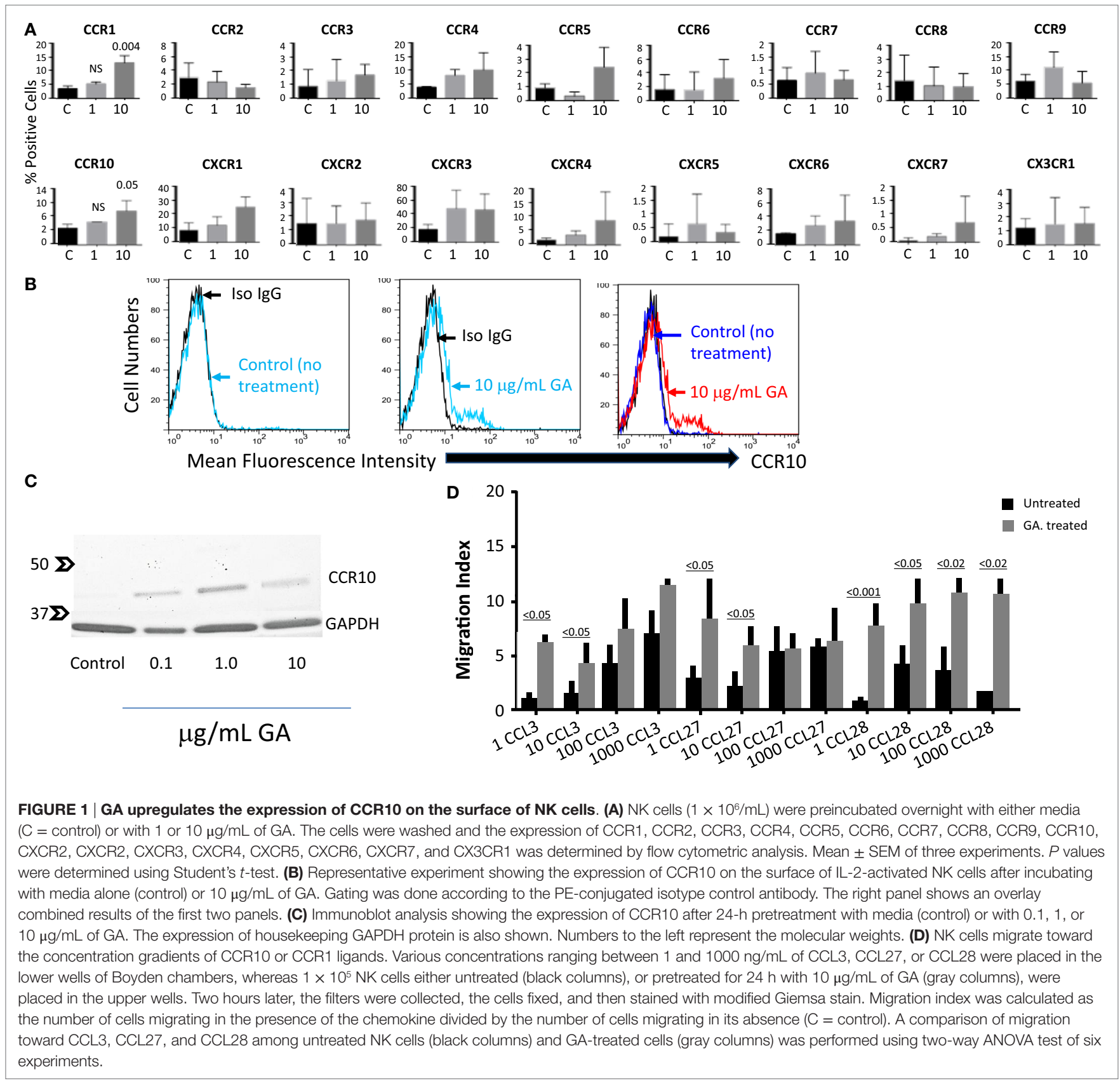

although a trend in increased release of CCL28 was noted after pretreatment with $10 \mu \mathrm{g} / \mathrm{mL}$ GA (Figure S2 in Supplementary Material).

Next, we examined whether GA or a combination of GA with CCL27 or CCL28 might induce the expression of CD107a on the surface of activated NK cells. Histograms of flow cytometric analysis showed a trend of increased CD107a expression after incubating the cells with GA, CCL27, or CCL28 (Figure S2C in Supplementary Material, left). Combining GA with CCL27 or CCL28 did not augment such expression when compared to cells incubated with GA alone (Figure S2C in Supplementary Material, right). When three or more experiments were performed, the trend of increased expression of CD107a was also noticed after incubating the cells with GA, CCL27, or CCL28. However, such enhancement did not reach significant levels after treating the cells with GA, any of the two chemokines, or GA plus the chemokines (Figure S2D in Supplementary Material).

\section{GA or Supernatants Collected from IL-2- Activated NK Cells Induce the Expression of Granzyme B in NK Cells: Inhibition by Anti-CCL28}

To further investigate the plausible mechanisms of CCR10/ chemokines axis, we measured the expression of Granzyme B in activated NK cells. For this, NK cells were either incubated with 


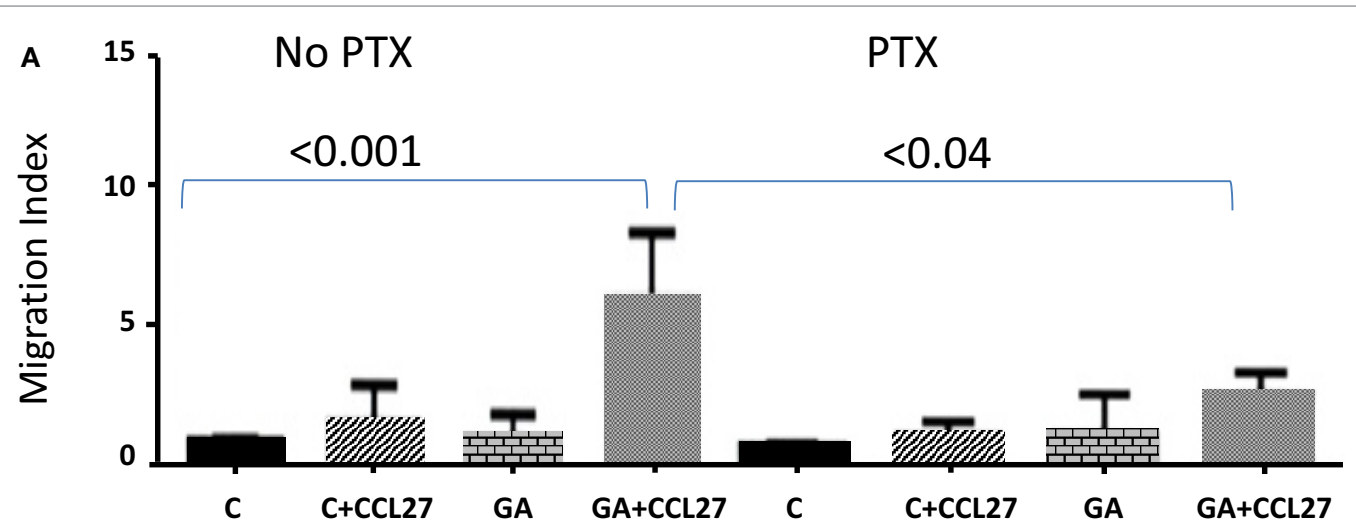

в $\quad$ No PTX

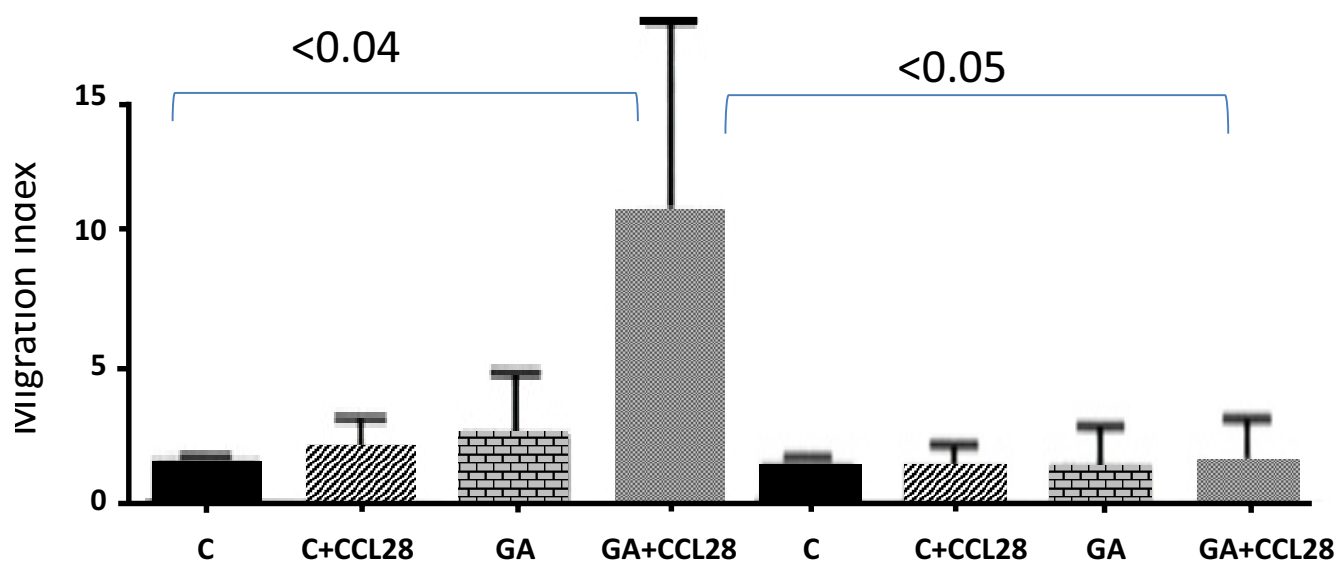

FIGURE 2 | Pretreatment with pertussis toxin (PTX) inhibits GA activity. (A) Migration of cells either left untreated (C = control) or pretreated overnight with $10 \mu \mathrm{g} / \mathrm{mL}$ of GA toward $10 \mathrm{ng} / \mathrm{mL}$ CCL27. Before the assay, these cells were either left intact (left columns) or intoxicated with $100 \mathrm{ng} / \mathrm{mL}$ of PTX for $2 \mathrm{~h}$ (right columns). (B) Migration of cells either untreated (C = control) or pretreated overnight with $10 \mu \mathrm{g} / \mathrm{mL}$ of GA toward $1 \mathrm{ng} / \mathrm{mL}$ CCL28. Before the assay, these cells were either left intact (left columns) or intoxicated with $100 \mathrm{ng} / \mathrm{mL}$ of PTX for $2 \mathrm{~h}$ (right columns). $P$ values comparing the migration of cells toward chemokines, and its inhibition by pretreatment with PTX, are determined by two-way ANOVA test of six different experiments. $\mathrm{C}=\mathrm{control}$. C+ chemokine $=$ untreated cells migrating toward the chemokine. GA = cells treated with GA overnight and migrated toward media only (no chemokine). GA+ chemokine = cells treated with GA overnight and then migrated toward the chemokine.

media alone or with $10 \mu \mathrm{g} / \mathrm{mL}$ of GA overnight, and the expression of Granzyme (GrB) was detected by flow cytometric analysis. Such treatment increased the percentages of cells expressing $\mathrm{GrB}$ from 6 to about 30\% after pretreatment with the drug (Figure S3 in Supplementary Material). Supernatants collected from activated NK cells, when added to NK cells, also increased such expression to about $12 \%$. The enhancement of GrB expression by the supernatants was lowered by pretreatment with anti-CCL28 to about $6 \%$, but not with anti-CCL27, whereas the isotype control for the anti-CCL27 and anti-CCL28 also did not affect the activity of the supernatants (Figure S3 in Supplementary Material, upper panels). Addition of the supernatants to GA did not result in any synergy among their activities. However, antiCCL28, and to a lesser extent anti-CCL27, reduced the effect of GA on GrB expression (Figure S3 in Supplementary Material, lower panels).

\section{DMF or MMF Also Upregulates the Expression of CCR10 on the Surface of IL-2-Activated NK Cells and Induces Their Chemotaxis}

To determine whether other drugs or metabolites used to treat or have potential for treating MS or cancer patients might affect the expression of CCR10 on NK cells similar to GA, we investigated the effects of various drugs. We observed that none of the concentrations of vitamin $\mathrm{D}_{3}$, its metabolite Calcipotriol, or FTY720 upregulated the expression of CCR10 on the surface of activated NK cells (not shown). In contrast, two different concentrations of MMF ( 1 and $100 \mu \mathrm{M}$ ) or the $100 \mu \mathrm{M}$ concentration of DMF significantly upregulated the expression of CCR10 on the surfaces of NK cells (Figure 4A). MMF and the drug DMF also upregulated the expression of CXCR3, but not any other 

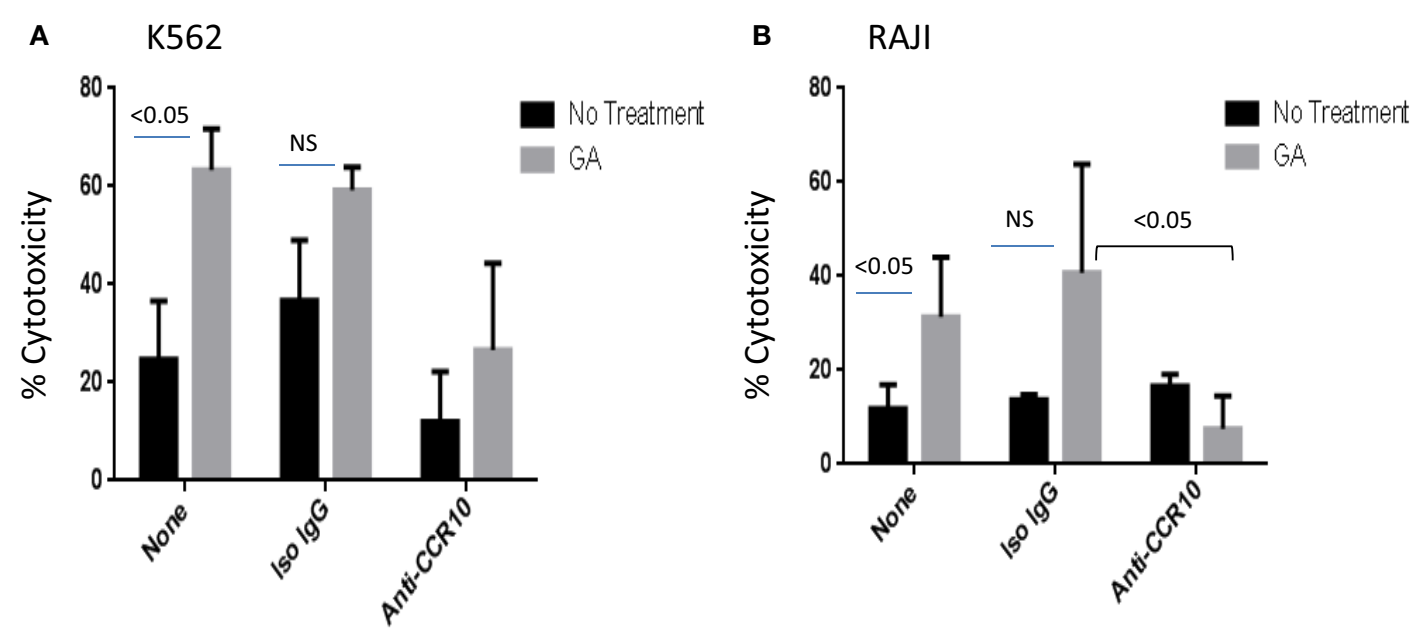

FIGURE 3 | Anti-CCR10 inhibits NK cell lysis of tumor target cells. (A) NK cells were either left untreated (black columns) or incubated with $10 \mu \mathrm{g} / \mathrm{mL}$ of GA overnight (gray columns). Before the assay the cells were incubated for 45 min with isotype control lgG antibody or with $10 \mu \mathrm{g} / \mathrm{mL}$ of anti-CCR10, washed, and then mixed with Calcein-AM-labeled K562 cells in the 4-h NK cytotoxicity assay. (B) This is similar to (A) except that RAJl tumor cells were used as targets instead of K562 cells. Mean \pm SEM of four separate experiments.

chemokine receptor examined (Figure 4A). Histograms of one representative experiment showed that 1 and $100 \mu \mathrm{M}$ of $\mathrm{MMF}$ or $100 \mu \mathrm{M}$ of DMF upregulated the expression of CCR10 on the surface of human IL-2-activated NK cells when compared to cells unstimulated with the drugs (Figure 4B). To corroborate the expression of CCR10 with functional activity, we performed the chemotaxis assay. Treatment of NK cells with MMF for $24 \mathrm{~h}$ enhanced their chemotaxis toward CCL27, CCL28, and CXCL10, the ligand for CXCR3 (Figure 4C). These results correlated well with increased expression of CCR10 and CXCR3 on NK cells after incubation with 1 or $100 \mu \mathrm{M}$ MMF. Surprisingly, pretreatment of IL-2-activated NK cells with DMF did not increase their chemotaxis toward CCL27. However, their chemotaxis was increased toward $1 \mu \mathrm{g} / \mathrm{mL}$ of CCL28 or CXCL10 after overnight incubation with $100 \mu \mathrm{M}$ DMF (Figure 4D).

\section{Anti-CCR10 Inhibits MMF or DMF-Induced NK Cell Lysis of Tumor Target Cells}

We recently reported that MMF enhanced freshly isolated NK cell lysis of tumor cells (20). Because of the differences in the expression of NK cytotoxicity receptors among naive vs. activated NK cells, we sought to investigate whether MMF or DMF might enhance activated NK cell lysis of tumor cells. Pretreatment with either MMF or DMF enhanced IL-2-activated NK cell killing of K562 tumor cells (Figure 5A). However, only pretreatment with MMF, and not DMF, augmented activated NK cell lysis of the B cell lymphoma RAJI cells (Figure 5B). These results mirrored our recent findings showing that MMF, and not DMF, increased lysis of naive NK cells against RAJI cells (20).

Next, we asked whether CCR10 might play a role in MMF or DMF augmentation of NK cell cytotoxicity. Similar to its effect on GA-enhanced NK cell cytotoxicity, incubating activated NK cells with anti-CCR10 for 45 min abrogated the cytotoxicity induced by MMF or DMF against K562 cells (Figure 5A) or MMFenhanced activated NK cell cytotoxicity against RAJI tumor cells (Figure 5B). This effect is not related to a general toxic effect of anti-CCR10 antibody as more than $95 \%$ of the cells were viable after treatment for $45 \mathrm{~min}$ with the antibodies.

\section{DISCUSSION}

Natural killer cells stand at the cross road among treatment of autoimmune diseases and immunodeficient diseases. In autoimmune diseases, the best protocol is to suppress the immune system, which is achieved by immunosuppressive drugs. On the other hand, in immunodeficient diseases, such as cancer or AIDS, the immune system must be activated to fight cancer cells or virally infected cells, respectively. From a first look, it appears that these methods of treatment are contradictory to each other, i.e., immunosuppression vs. immunostimulation. However, NK cells can be used as a therapeutic modality for both autoimmune diseases and cancer (23). Although there are more than $600 \mathrm{NCI}$ approved clinical trials using NK cells (http://www.cancer.gov/ search/results) to treat various forms of cancer, investigators are faced with the problems of targeting these highly antitumor effectors toward the sites of tumor growth.

Given the fact that NK cells can kill tumors, several strategies for the therapeutic use of NK cells have been proposed and tried in a clinical context. Cytokines have been used in the treatment of some human cancers and, in some instances, the mechanisms of action are through direct or indirect activation of NK cells (24). NK cell differentiation and activation is affected by cytokines such as IL-2, IL-12, IL-15, IL-18, IL-21, and IFNs. Several clinical trials have assessed the effects of IL-2 administration on activation and expansion of NK cells in patients with cancer (25). Apart from specific cytokines and/or growth factors, broad activators of immune function that are used in cancer treatment may also act 


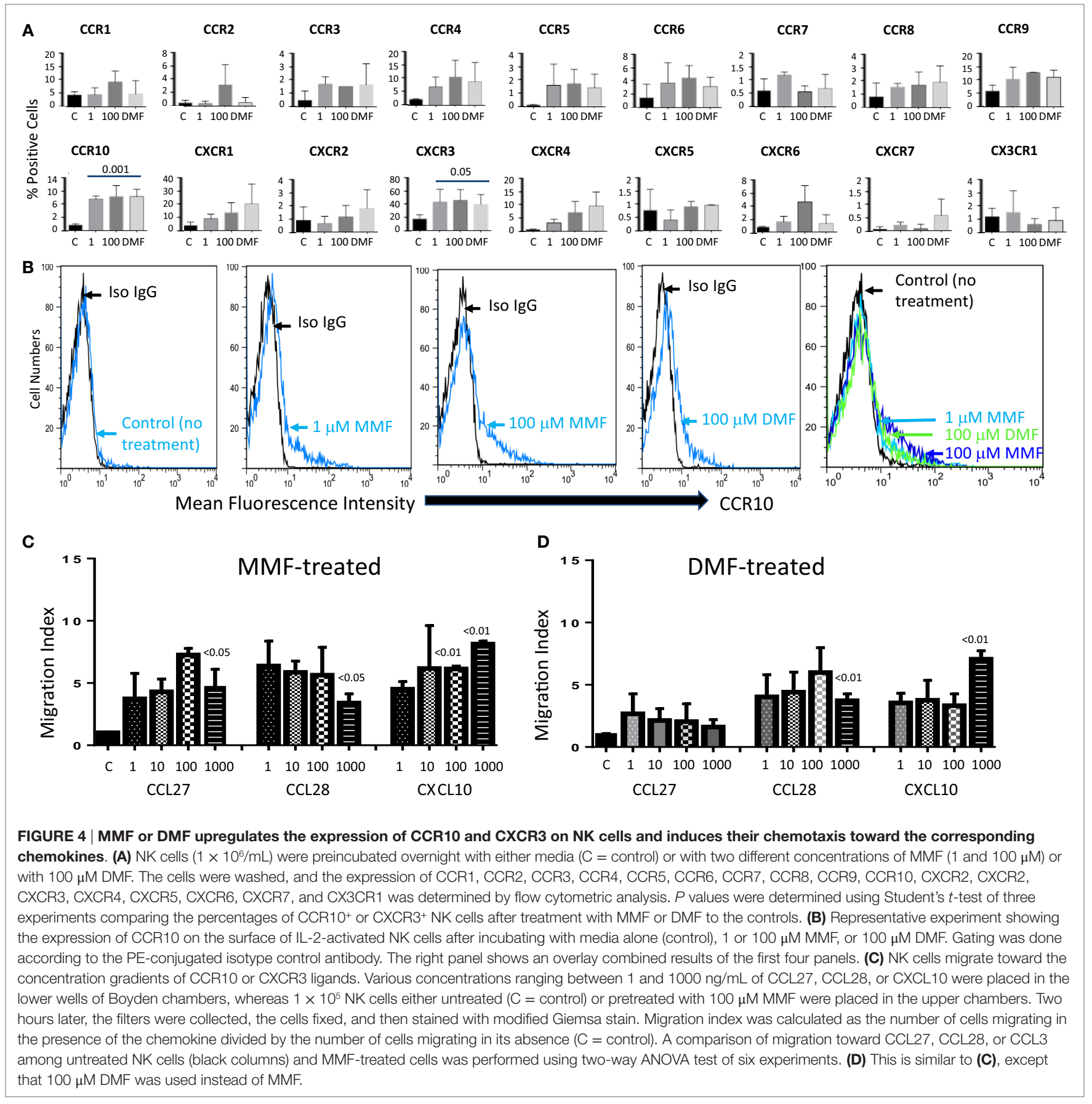

on NK cells (25). Similarly, NK cells may have a role in the clinical efficacy of Mycobacterium bovis bacillus Calmette-Guérin (BCG) treatment of bladder cancer (26), indicating that mediators, which activate endogenous $\mathrm{NK}$ cells, can induce immune-mediated control of cancer. The potential efficacy of NK cells in treating various forms of cancer has been extensively reviewed (27-33).

CCL27 (CTACK) is expressed in epidermal keratinocytes and is responsible for attracting CCR10 $10^{+} \mathrm{T}$ cells into the skin (34, 35). On the other hand, CCL28 is expressed by epithelial cells of mucosal sites (36-38) and is upregulated in inflamed tissues such as colon, duodenal mucosa, lungs, and liver ducts (39-41).
Further, treatment of Crohn's disease patients resulted in reduced expression of CCR10 corroborated with reduced inflammation in those patients [reviewed in Ref. (42)].

Intratumoral administration of CCR10 as well as other chemokine-expressing adenoviral vector into tumor-bearing animals resulted in the recruitment and activation of $\mathrm{T}$ cells at sites of tumor growth (43). Similarly, transfecting CCL27 into ovarian carcinoma cells resulted in reduced tumor growth by antitumor immune responses (44). Human keratinocyte-derived skin tumors can evade T cells antitumor activity by downregulating the expression of CCR10. Further, in vivo work demonstrated that 

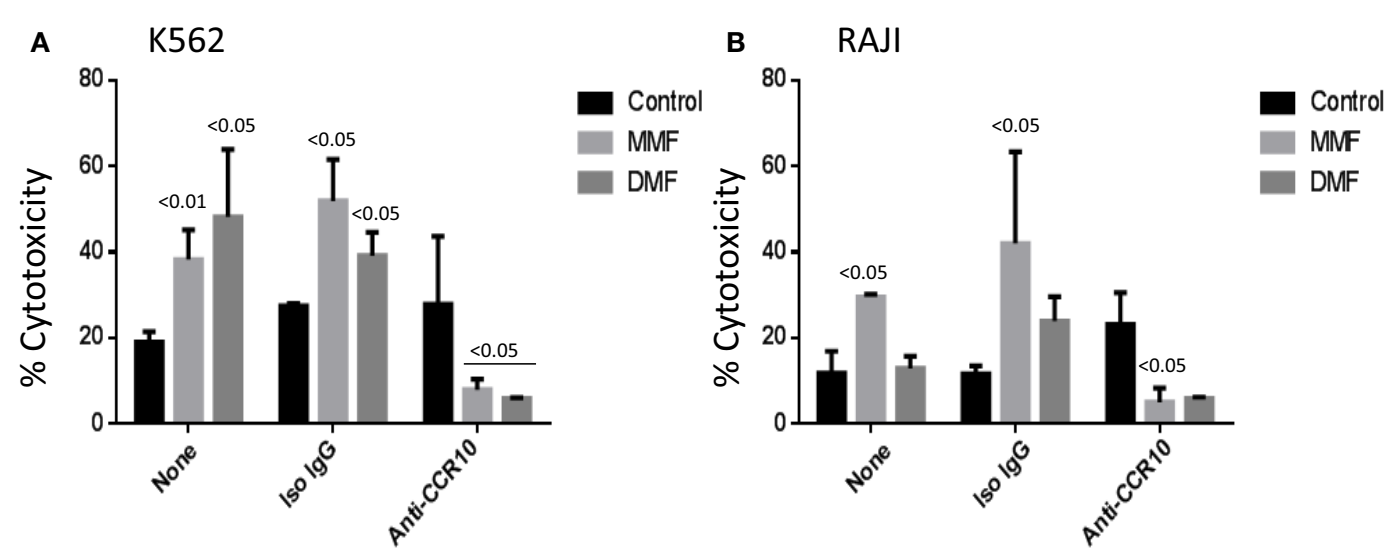

FIGURE 5 | MMF or DMF augments activated NK cell cytotoxicity against tumor cells: inhibition by anti-CCR10 antibody. (A) NK cells were either left intact (black columns) or incubated with $100 \mu \mathrm{M}$ MMF or DMF overnight (gray columns), washed, and then incubated with K562 cells. (B) is similar to (A) except that RAJl cells were used in the NK cytotoxicity assay. For treatment with anti-CCR10, the cells were washed and incubated with either $10 \mu \mathrm{g} / \mathrm{mL}$ of anti-CCR10 or isotype control lgG antibody for $45 \mathrm{~min}$ before incubating with the target cells. Percent cytotoxicity was then measured, and significant values as compared to the control in each treatment are shown on top of the columns. Significance of cytotoxicity in the presence of anti-CCR10 was compared to the cytotoxicity in the presence of isotype control IgG antibody. Mean \pm SEM of four experiments.

neutralization of CCL27 decreased leukocyte recruitment toward cutaneous tumor sites, resulting in enhanced tumor growth (45).

In contrast, it was observed that overexpression of CCR 10 and CCR7 resulted in severe outcome of human cutaneous melanoma growth, as determined by high risk of relapse and death of patients (46). Along these lines, Kai et al. (47) observed that CCR10 and CCL27 were strongly expressed in human cutaneous squamous cell carcinoma, which is advantageous for tumor cell survival and proliferation. Further, hypoxic tumor cells released CCL28 recruiting CCR $10^{+}$Treg cells, which promote tumor growth and angiogenesis, an activity that was abrogated by anti-CCL28 (48).

To this end, we observed that GA upregulates the expression of CCR10 on the surface of human NK cells. This activity corroborated with enhanced migration of these cells toward the concentration gradients of CCL27 and CCL28, the ligands for CCR10. PTX inhibited the chemotactic effects induced by chemokines, suggesting that $G$ protein-coupled receptors (GPCRs), and in particular $\mathrm{G}_{\mathrm{i} / \mathrm{o}}$, are involved. PTX also inhibited the cytolytic activity of NK cells induced by GA against tumor cells. Because chemokines bind GPCRs, we entertained the possibility that CCR10 could be involved in mediating NK cell cytotoxicity. We observed that anti-CCR10 reduced NK cell lysis of tumor target cells. Although the reasons behind such enigmatic effect are not clear, it is plausible that a complex interaction takes place resulting in activating the lytic potential of NK cells. It is conceivable that CCR10 upregulated by GA might interact with the CCR10 ligands such as CCL27 and CCL28 secreted by activated NK cells. Such binding may result in inducing intracellular signaling molecules that may mediate the cytolytic activity of NK cells. Hence, antiCCR10 or PTX might interfere with such interaction resulting in inhibiting NK cells mediating lysis of tumor cells. Our results did not detect any increases in the levels of CCL27 or CCL28 secreted by NK cells after stimulation with GA. Neither there was a synergy among GA and these chemokines in the upregulation of CD107a on the surface of these cells. This molecule is considered an activation marker for NK cell-mediated lysis of tumor cells, and its expression is increased after incubating resting NK cells with MMF (20). Further analysis showed that supernatants collected from activated NK cells increased the percentages of Granzyme B positive cells, and that such activity was inhibited by pretreatment with anti-CCL28 indicating that CCL28 secreted by activated NK cells might bind CCR10 and consequently, upregulate the expression of lytic molecules on higher numbers of NK cells. GA also increased the percentages of Granzyme B positive NK cells, and this effect was reduced by anti-CCL28. Albeit not examined, it is plausible that GA might also utilize CCL28, perhaps to increase the expression of GrB. Although we have not seen a significant increase of CCL28 release after pretreatment with GA, we noted a trend of such enhancement.

Similar to GA, MMF and DMF also upregulated the expression of CCR10 on the surface of activated NK cells. Further, these chemicals enhanced activated NK cell lysis of tumor target cells. Similar to GA effect, such enhancement of cytotoxicity was abrogated by pretreatment with anti-CCR10. More work is needed to explore in details such an exciting possibility involving chemokines/chemokine receptors axis in mediating not only the migration of NK cells but also other activities such as cytotoxicity.

We consider the most important aspect of this work is the finding that drugs for MS such as GA and DMF as well as MMF upregulate the expression of CCR10 on the surface of IL-2-activated NK cells. Such finding might have potential relevance for treating cancer, particularly those tumors that secrete chemokines, which bind CCR10, including malignant melanomas and squamous cell carcinomas, which secrete CCL27 $(47,49)$, or colorectal cancer cells that secrete CCL28 (50). This is particularly relevant when one considers that there is an estimated $2-5 \times 10^{9} \mathrm{NK}$ cells in $5 \mathrm{~L}$ of blood. A $1 \%$ of this NK cell number represents a subset of NK cells that expresses CCR10 (an estimated number of $20-50 \times 10^{8} / 5 \mathrm{~L}$ of blood). A $10 \%$ increase after treatment with the drugs described in this manuscript suggests that the number of CCR $10^{+} \mathrm{NK}$ cells 
might be increased to about $200-500 \times 10^{8} / 5 \mathrm{~L}$ of blood; a substantial number of killer cells that may potentially inhibit tumor growth. Our next step is to examine in tumor-bearing animals whether these CCR $10^{+}$-activated killer cells may migrate into the sites of colorectal cancers or melanomas after pretreatment with GA, MMF, or DMF. A number of cancers are, at present, incurable. For others, chemotherapy is only partially effective, and a significant proportion of patients relapse following treatment. Some hematological malignancies are treatable by hematopoietic stem cell transplantation (HSCT), but fewer than $30 \%$ of patients requiring HSCT have a suitable donor. NK cells possess the ability to spontaneously lyse certain target cells, including tumor cells. The major issue that faced investigators in this field is the inability of NK cells to migrate toward sites of tumor growth. This, despite the existence of more than 600 approved clinical trials using NK cells to treat various cancers. Our present work may provide a novel approach of harnessing activated NK cells in vitro for the purpose of administering into cancer patients. We propose that these cells can be harnessed in vitro with drugs such as GA, DMF (or MMF). These drugs perform two important functions for these cells; first, they increased their cytotoxicity against tumor cells, and second, they upregulate the expression of CCR10 on their surfaces. Consequently, NK cells can be targeted toward the growth of tumor cells that secrete CCL27 and CCL28.

\section{CONCLUSION}

We previously described the effects of GA on chemokine receptor expression in an MS patient receiving this drug (51). However, this is the first demonstration that GA, DMF, and MMF upregulate the expression of an important chemokine receptor, i.e., CCR10, on the surface of activated NK cells. Such activity might have important implications in NK cell immunotherapy. Because immunotherapy using NK cells suffers from the inability of these cells to migrate into tumor stroma, it is now feasible to direct these cells toward sites of tumor growth, particularly those secreting CCL27 and CCL28. Hence, we suggest the feasibility of using such approach to treat cutaneous squamous cell carcinomas and squamous melanoma, as well as colorectal cancer.

\section{REFERENCES}

1. Cooper MA, Fehniger TA, Caligiuri MA. The biology of human natural killer-cell subsets. Trends Immunol (2001) 22:633-40. doi:10.1016/S14714906(01)02060-9

2. Moretta A. Natural killer cells and dendritic cells: rendezvous in abused tissues. Nat Rev Immunol (2002) 2:957-64. doi:10.1038/nri956

3. Biron CA, Nguyen KB, Pien GC, Cousens LP, Salazar-Mather TP. Natural killer cells in antiviral defense: function and regulation by innate cytokines. Ann Rev Immunol (1999) 17:189-220. doi:10.1146/annurev. immunol.17.1.189

4. Maghazachi AA, Al-Aoukaty A. Chemokines activate natural killer cells through heterotrimeric G-proteins: implications for the treatment of AIDS and cancer. FASEB J (1998) 12:913-24.

5. Maghazachi AA. Insights into seven and single transmembrane-spanning domain receptors and their signaling pathways in human natural killer celles. Pharmacol Rev (2005) 57:339-57. doi:10.1124/pr.57.3.5

6. Imai K, Matsuyama S, Miyake S, Suga K, Nakachi K. Natural cytotoxic activity of peripheral-blood lymphocytes and cancer incidence: an 11-year follow-up study of a general population. Lancet (2000) 356:1795-9. doi:10.1016/S01406736(00)03231-1

\section{AUTHOR CONTRIBUTIONS}

AAM designed the experiments, performed the chemotaxis assays, analyzed the data, and wrote the paper. KS and ZA-J performed the flow cytometric experiments and other assays.

\section{SUPPLEMENTARY MATERIAL}

The Supplementary Material for this article can be found online at http://journal.frontiersin.org/article/10.3389/fimmu.2016.00437

FIGURE S1 | Expression of CD56 molecule on the surface of IL-2-activated NK cells. (A) Activated NK cells were labeled with either mouse FITC-conjugated isotype IgG or mouse FITC-conjugated anti-CD3 antibody. (B) Activated NK cells labeled with mouse PE-conjugated isotype IgG or mouse PE-conjugated anti-CD56 antibody. (C) Activated NK cells labeled with both FITC-conjugated anti-CD3 antibody and PE-conjugated anti-CD56 and examined in the flow cytometry. One of the four representative experiments was performed.

FIGURE S2 | IL-2-activated NK cells release CCL27 and CCL28 and express CD107a. (A) NK cells were either left intact or were incubated for $24 \mathrm{~h}$ with $0.1,1$ or $10 \mu \mathrm{g} / \mathrm{mL}$ GA. Supernatants were collected, and the levels of CCL27 were measured. (B) CCL28 levels were evaluated using ELISA assay. (C) A representative experiment showing histograms for the expression of CD107a after incubating overnight with $10 \mu \mathrm{g} / \mathrm{mL}$ GA, $10 \mathrm{ng} / \mathrm{mL}$ CCL27, or $10 \mathrm{ng} / \mathrm{mL}$ CCL28 (left panel), or $10 \mu \mathrm{g} / \mathrm{mL}$ GA either alone or in combination with $10 \mathrm{ng} / \mathrm{mL}$ CCL27 or $10 \mathrm{ng} / \mathrm{mL}$ CCL28 (right panel). (D) Activated NK cells $(1 \times 10 \% \mathrm{~mL})$ were either untreated (-) or incubated overnight with $10 \mu \mathrm{g} / \mathrm{mL}$ GA (GA), $100 \mathrm{ng} /$ $\mathrm{mL}$ CCL27, $100 \mathrm{ng} / \mathrm{mL}$ CCL28, or a combination of $10 \mu \mathrm{g} / \mathrm{mL}$ GA with $100 \mathrm{ng} /$ $\mathrm{mL}$ CCL27 (GA + CCL27), or $100 \mathrm{ng} / \mathrm{mL}$ CCL28 (GA + CCL28). The cells were washed, and the expression of CD107a was evaluated by flow cytometric analysis. Mean \pm SEM of values from three different donors. The binding of isotype control antibody is also shown.

FIGURE S3 | GA or supernatants collected from IL-2-activated NK cells increase the percentages of NK cells expressing Granzyme B. IL-2activated NK cells were incubated for $24 \mathrm{~h}$ with either media alone or with $10 \mu \mathrm{g} / \mathrm{mL}$ GA. In both treatments, the cells were incubated with supernatants collected from IL-2-activated NK cells. In other cultures, the cells were pretreated with the supernatants in the presence of $1 \mu \mathrm{g} / \mathrm{mL}$ mouse $\mathrm{lgG}$ isotype control for anti-CCL27 and anti-CCL28, or with $1 \mu \mathrm{g} / \mathrm{mL}$ of neutralizing mouse anti-CCL27 or mouse anti-CCL28. Upper panels show expression of Granzyme B in the absence of GA, whereas lower panels show expression of the same molecule in the presence of $10 \mu \mathrm{g} / \mathrm{mL}$ GA. One of the two representative experiments was performed. Percentages of positive cells are shown between brackets.

7. Ishigami S, Natsugoe S, Tokuda K, Nakajo A, Che X, Iwashige H, et al. Prognostic value of intratumoral natural killer cells in gastric carcinoma. Cancer (2000) 88:577-83. doi:10.1002/(SICI)1097-0142(20000201)88:3<577::AIDCNCR13>3.3.CO;2-M

8. Luster AD. The role of chemokines in linking innate and adaptive immunity. Curr Opin Immunol (2002) 14:29-35.

9. Raman D, Sobolik-Delmaire T, Richmond A. Chemokines in health and disease. Exp Cell Res (2011) 317:575-89. doi:10.1016/j.yexcr.2011.01.005

10. Sebok K, Woodside D, al-Aoukaty A, Ho AD, Gluck S, Maghazachi AA. IL-8 induces the locomotion of human IL-2-activated natural killer cells. Involvement of a guanine nucleotide binding (Go) protein. J Immunol (1993) 150:1524-34.

11. Maghazachi AA, al-Aoukaty A, Schall TJ. C-C chemokines induce the chemotaxis of NK and IL-2-activated NK celles. Role for G proteins. J Immunol (1994) 153:4969-77.

12. Maghazachi AA. Role of chemokines in the biology of natural killer celles. Curr Top Microbiol Immunol (2010) 341:37-58.

13. Arnon R, Aharoni R. Mechanism of action of glatiramer acetate in multiple sclerosis and its potential for the development of new applications. Proc Natl Acad Sci U S A (2004) 101:14593-8. doi:10.1073/pnas. 0404887101 
14. Kala K, Miravalle A, Vollmer T. Recent insights into the mechanism of action of glatiramer acetate. J Neuroimmunol (2011) 235:9-17. doi:10.1016/j. jneuroim.2011.01.009

15. Ramtahal J, Jacob A, Das K, Boggild M. Sequential maintenance treatment with glatiramer acetate after mitoxantrone is safe and can limit exposure to immunosuppression in very active, relapsing remitting multiple sclerosis. J Neurol (2006) 253:1160-4. doi:10.1007/s00415-006-0178-Z

16. Sand KL, Knudsen E, Rolin J, Al-Falahi Y, Maghazachi AA. Modulation of natural killer cell cytotoxicity and cytokine release by the drug glatiramer acetate. Cell Mol Life Sci (2009) 66:1446-56. doi:10.1007/s00018-009-8726-1

17. Al-Falahi Y, Sand KL, Knudsen E, Damaj BB, Rolin J, Maghazachi AA. Splenic natural killer cell activity in two models of experimental neurodegenerative diseases. J Cell Mol Med (2009) 13:2693-703. doi:10.1111/j.1582-4934.2008. 00640.x

18. Al-Jaderi Z, Maghazachi AA. Utilization of dimethyl fumarate and related molecules for treatment of multiple sclerosis, cancer, and other diseases. Front Immunol (2016) 367:278. doi:10.3389/fimmu.2016.00278

19. Loewe R, Valero T, Kremling S, Pratscher B, Kunstfeld R, Pehamberger H, et al. Dimethylfumarate impairs melanoma growth and metastasis. Cancer Res (2006) 66:11888-96. doi:10.1158/0008-5472.CAN-06-2397

20. Vego H, Sand KL, Hoglund RA, Fallang LE, Gundersen G, Holmoy T, et al. Monomethyl fumarate augments NK cell lysis of tumor cells through degranulation and the upregulation of NKp46 and CD107a. Cell Mol Immunol (2016) 13:57-64. doi:10.1038/cmi.2014.114

21. Al-Jaderi Z, Maghazachi AA. Vitamin D3 and monomethyl fumarate enhance natural killer cell lysis of dendritic cells and ameliorate the clinical score in mice suffering from experimental autoimmune encephalomyelitis. Toxins (2015) 7:4730-44. doi:10.3390/toxins7114730

22. Maghazachi AA, Skalhegg BS, Rolstad B, Al-Aoukaty A. Interferon-inducible protein-10 and lymphotactin induce the chemotaxis and mobilization of intracellular calcium in natural killer cells through pertussis toxin-sensitive and -insensitive heterotrimeric G-proteins. FASEB J (1997) 11:765-74.

23. Maghazachi AA. Role of natural killer cells in multiple sclerosis. ISRN Immunol (2012) 2012:1-14. doi:10.5402/2012/795075

24. Farag SS, Caligiuri MA. Cytokine modulation of the innate immune system in the treatment of leukemia and lymphoma. Adv Pharmacol (2004) 5:295-318. doi:10.1016/S1054-3589(04)51013-X

25. Rosenberg SA. Interleukin-2 and the development of immunotherapy for the treatment of patients with cancer. Cancer J Sci Am (2000) 6(Suppl 1):2-7.

26. Gomes-Giacoia E, Miyake M, Goodison S, Sriharan A, Zhang G, You L, et al. Intravesical ALT-803 and BCG treatment reduces tumor burden in a carcinogen induced bladder cancer rat model; a role for cytokine production and NK cell expansion. PLoS One (2014) 9:e96705. doi:10.1371/journal.pone. 0096705

27. Rezvani K, Rouce RH. The application of natural killer cell immunotherapy for the treatment of cancer. Front Immunol (2015) 6:578. doi:10.3389/ fimmu.2015.00578

28. Carotta S. Targeting NK cells for anticancer immunotherapy: clinical and preclinical approaches. Front Immunol (2016) 7:152. doi:10.3389/fimmu. 2016.00152

29. Iannello A, Thompson TW, Ardolino M, Marcus A, Raulet DH. Immunosurveillance and immunotherapy of tumors by innate immune cells. Curr Opin Immunol (2016) 38:52-8. doi:10.1016/j.coi.2015.11.001

30. Dahlberg CI, Sarhan D, Chrobok M, Duru AD, Alici E. Natural killer cell-based therapies targeting cancer: possible strategies to gain and sustain anti-tumor activity. Front Immunol (2015) 6:605. doi:10.3389/fimmu.2015.00605

31. Davis ZB, Felices M, Verneris MR, Miller JS. Natural killer cell adoptive transfer therapy: exploiting the first line of defense against cancer. Cancer $J$ (2015) 6:486-91. doi:10.1097/PPO.0000000000000156

32. Berrien-Elliott MM, Romee R, Fehniger TA. Improving natural killer cell cancer immunotherapy. Curr Opin Organ Transplant (2015) 20:671-80. doi:10.1097/MOT.0000000000000243

33. Cantoni C, Grauwet K, Pietra G, Parodi M, Mingari MC, Maria AD, et al. Role of NK cells in immunotherapy and virotherapy of solid tumors. Immunotherapy (2015) 7:861-82. doi:10.2217/imt.15.53

34. Morales J, Homey B, Vicari AP, Hudak S, Oldham E, Hedrick J, et al. CTACK, a skin-associated chemokine that preferentially attracts skin-homing memory T cells. Proc Natl Acad Sci U S A (1999) 96:14470-5. doi:10.1073/ pnas.96.25.14470
35. Hudak S, Hagen M, Liu Y, Catron D, Oldham E, McEvoy LM, et al. Immune surveillance and effector functions of CCR10(+) skin homing $\mathrm{T}$ cells. J Immunol (2002) 169:1189-96. doi:10.4049/jimmunol.169.3.1189

36. Pan J, Kunkel EJ, Gosslar U, Lazarus N, Langdon P, Broadwell K, et al. A novel chemokine ligand for CCR10 and CCR3 expressed by epithelial cells in mucosal tissues. J Immunol (2000) 165:2943-9. doi:10.4049/jimmunol.165.6.2943

37. Wang W, Soto H, Oldham ER, Buchanan ME, Homey B, Catron D, et al. Identification of a novel chemokine (CCL28), which binds CCR10 (GPR2). J Biol Chem (2000) 275:22313-23. doi:10.1074/jbc.M001461200

38. Hieshima K, Ohtani H, Shibano M, Izawa D, Nakayama T, Kawasaki Y, et al. CCL28 has dual roles in mucosal immunity as a chemokine with broadspectrum antimicrobial activity. J Immunol (2003) 170:1452-61. doi:10.4049/ jimmunol.170.3.1452

39. Ogawa H, Iimura M, Eckmann L, Kagnoff MF. Regulated production of the chemokine CCL28 in human colon epithelium. Am J Physiol Gastrointest Liver Physiol (2004) 287:G1062-9. doi:10.1152/ajpgi.00162.2004

40. Maeda S, Ohno K, Tsukamoto A, Nakashima K, Fukushima K, Goto-Koshino $\mathrm{Y}$, et al. Molecular cloning and expression analysis of the canine chemokine receptor CCR9. Vet Immunol Immunopathol (2011) 144:290-8. doi:10.1016/j. vetimm.2011.08.020

41. Scanlon KM, Hawksworth RJ, Lane SJ, Mahon BP. IL-17A induces CCL28, supporting the chemotaxis of IgE-secreting B cells. Int Arch Allergy Immunol (2011) 156:51-61. doi:10.1159/000322178

42. Xiong N, Fu Y, Hu S, Xia M, Yang J. CCR10 and its ligands in regulation of epithelial immunity and diseases. Protein Cell (2012) 3:571-80. doi:10.1007/ s13238-012-2927-3

43. Okada N, Sasaki A, Niwa M, Okada Y, Hatanaka Y, Tani Y, et al. Tumor suppressive efficacy through augmentation of tumor-infiltrating immune cells by intratumoral injection of chemokine-expressing adenoviral vector. Cancer Gene Ther (2006) 13:393-405. doi:10.1038/sj.cgt.7700903

44. Okada N, Gao JQ, Sasaki A, Niwa M, Okada Y, Nakayama T, et al. Anti-tumor activity of chemokine is affected by both kinds of tumors and the activation state of the host's immune system: implications for chemokine-based cancer immunotherapy. Biochem Biophys Res Commun (2004) 317:68-76. doi:10.1016/ j.bbrc.2004.03.013

45. Pivarcsi A, Müller A, Hippe A, Rieker J, van Lierop A, Steinhoff M, et al. Tumor immune escape by the loss of homeostatic chemokine expression. Proc Natl Acad Sci U S A (2007) 104:19055-60. doi:10.1073/pnas.0705673104

46. Kühnelt-Leddihn L, Müller H, Eisendle K, Zelger B, Weinlich G. Overexpression of the chemokine receptors CXCR4, CCR7, CCR9, and CCR10 in human primary cutaneous melanoma: a potential prognostic value for CCR7 and CCR10? Arch Dermatol Res (2012) 304:185-93. doi:10.1007/ s00403-012-1222-8

47. Kai H, Kadono T, Kakinuma T, Tomita M, Ohmatsu H, Asano Y, et al. CCR10 and CCL27 are overexpressed in cutaneous squamous cell carcinoma. Pathol Res Pract (2011) 207:43-8. doi:10.1016/j.prp.2010.10.007

48. Facciabene A, Peng X, Hagemann IS, Balint K, Barchetti A, Wang LP, et al. Tumour hypoxia promotes tolerance and angiogenesis via CCL28 and T(reg) cells. Nature (2011) 475:226-30. doi:10.1038/nature10169

49. Ben-Baruch A. Organ selectivity in metastasis: regulation by chemokines and their receptors. Clin Exp Metastasis (2008) 25:345-56. doi:10.1007/ s10585-007-9097-3

50. Dimberg J, Hugander A, Wågsäter D. Protein expression of the chemokine, CCL28, in human colorectal cancer. Int J Oncol (2006) 28:315-9.

51. Høglund RA, Hestvik AL, Holmøy T, Maghazachi AA. Expression and functional activity of chemokine receptors in glatiramer acetate-specific $\mathrm{T}$ cells isolated from multiple sclerosis patient receiving the drug glatiramer acetate. Hum Immunol (2011) 72:124-34. doi:10.1016/j.humimm.2010.10.016

Conflict of Interest Statement: The authors declare that the research was conducted in the absence of any commercial or financial relationships that could be construed as a potential conflict of interest.

Copyright (C) 2016 Maghazachi, Sand and Al-Jaderi. This is an open-access article distributed under the terms of the Creative Commons Attribution License (CC BY). The use, distribution or reproduction in other forums is permitted, provided the original author(s) or licensor are credited and that the original publication in this journal is cited, in accordance with accepted academic practice. No use, distribution or reproduction is permitted which does not comply with these terms. 